\title{
APPLICATION OF COST MATRICES AND COST CURVES TO ENHANCE DIAGNOSTIC HEALTH MANAGEMENT METRICS FOR GAS TURBINE ENGINES
}

\author{
Craig R. Davison
}

\author{
Gas Turbine Laboratory \\ Institute for Aerospace Research \\ National Research Council Canada \\ Ottawa, Ontario, Canada
}

\begin{abstract}
Statistically based metrics for gas turbine engine diagnostic systems are required to evaluate competing products fairly and to establish a convincing business case. Diagnostic algorithm validation often includes engine testing with implanted faults. The implantation rate is rarely, if ever, representative of the true fault occurrence rate. A technique is presented to convert a confusion matrix with a non-representative fault distribution to one representative of the expected distribution. The small sample size associated with fault implantation studies requires a confidence interval on the results to provide valid comparisons and a method for calculating them is presented.

The use of cost matrices to weight confusion matrices, based on the associated cost of each outcome, is demonstrated. The calculation of metrics on the resulting weighted confusion matrix will measure the relative cost of the diagnostic technique, rather than just its accuracy. In this paper, the generation and use of cost matrices is presented and their application to a hypothetical test case is demonstrated.

Receiver operating characteristic (ROC) curves evaluate diagnostic system performance across a range of threshold settings. This allows an algorithm's ability to be assessed over a range of possible usage. Cost curves are analogous to ROC curves but offer several advantages. The techniques for applying cost curves to diagnostic algorithms are presented and their advantages over ROC curves are outlined.
\end{abstract}

\section{INTRODUCTION}

In the past 10 years, interest has grown in defining statistically based suites of metrics for gas turbine engine diagnostic systems. As more diagnostic systems are developed, researchers require techniques to compare their algorithm to others. Organizations purchasing or funding research require metrics to evaluate competing products on a level playing field

\author{
Institute for Information Technology \\ National Research Council Canada \\ Ottawa, Ontario, Canada
}

\author{
Chris Drummond
}

and to establish a convincing business case. Davison and Bird [1] provided an overview of diagnostic metrics and proposed a technique to generate confidence intervals for them.

Diagnostic algorithm development is being performed by original equipment manufacturers (OEMs), small and medium businesses, research institutes and universities. While OEMs may have access to large quantities of operational data it is not always well correlated to the true state of the engine and access to these data sets by other developers can be extremely limited.

Developers often use results from computer engine models to test their algorithms. This technique can produce a wide range and large quantity of data, but the correlation to reality may be uncertain. Currently NASA is leading an initiative to produce a computer model which incorporates realistic fault implantation, operating condition variations and sensor errors. This will produce simulated operating data for a commercial aircraft engine for benchmarking diagnostic algorithm performance [2]. This will provide a useful generic tool for comparing algorithms, but may not be applicable to a particular user's operating conditions.

An alternate technique for algorithm testing uses data from implanted faults. While this is real engine data, it is usually acquired in a sea level test cell and is not representative of actual operating conditions. It also has a small sample size, due to the cost of performing such tests, resulting in a large uncertainty, which is often not quantified. This makes comparing algorithms difficult. Ideally the size of the confidence interval would be used to guide the required number of tests. However, as a minimum, the confidence interval corresponding to the sample size chosen should be presented. Failure to provide the confidence interval could result in a superior algorithm being discarded.

The fault distribution applied can also have a significant effect on an algorithms performance. During fault implantation 
studies the implantation rate is rarely, if ever, representative of the true fault occurrence rate. In addition, different operating environments result in different fault occurrence rates. The practical result being that an algorithm may have greater success in different operating environments, or on engines that generate different fault distributions. Modifying the confusion matrix to represent the expected fault distribution allows a more realistic assessment of the diagnostic algorithm under the expected operating conditions.

Similarly, the cost of fault occurrence and misdiagnosis impacts the algorithms value. A misdiagnosis with little effect on the bottom line will be a low priority for the end user, but a traditional confusion matrix does not account for this factor. Cost matrices allow algorithms to be compared based on such costs. They are easily applied to traditional confusion matrices allowing evaluation of a particular algorithm across a range of cost scenarios.

Receiver operating characteristic (ROC) curves suffer from many of the same problems as the traditional confusion matrix. This is not surprising as ROC curves are based on the decision matrix, which is a simple two case version of the confusion matrix. The ROC curve presents the decision matrix across a range of settings. Further details on ROC curves can be found throughout the literature, for example DePold et al. [3], and Davison and Bird [1], who examine them with relation to engine health, or Webb and Ting [4], who provide a broader discussion.

Cost curves have been presented by the artificial intelligence community as an improved technique for assessing algorithm performance across a range of settings. Cost curves present ROC data but over a range of fault distributions and costs [5,6]. This allows an algorithm to be assessed for applicability to a particular cost or fault distribution.

In addition, cost curves provide a simple visual comparison for two competing algorithms, and to the simple classifiers (all fault or all no fault). Cost curves allow simple averaging techniques to be applied to improve data quality, and confidence intervals to be generated for comparison purposes.

\section{NOMENCLATURE}

C Cost matrix

D Mean cost matrix

F Fault probability vector

F Number of fault types

FP False positive rate

L Laplace corrected confusion matrix

MSC Mean subjective cost score

NEC Normalized expected cost

P Confusion matrix as proportion of total diagnosis

PCC Percent correct classification

PCF Probability cost function

Q Confusion matrix normalized by fault proportion

TP True positive rate

U Upper limit

V Normalized cost matrix

$\mathrm{c}_{\mathrm{ij}} \quad$ Element in cost matrix $\mathrm{d}_{\mathrm{ij}} \quad$ Element in mean cost matrix

$\mathrm{f}_{\mathrm{i}} \quad$ Element in fault probability vector

$\mathrm{l}_{\mathrm{ij}} \quad$ Element in Laplace corrected confusion matrix

s Sample standard deviation

n Number of samples

$\mathrm{p}_{\mathrm{ij}} \quad$ Element in confusion matrix as proportion of total diagnosis

$\mathrm{p}_{+} \quad$ Proportion of fault cases

$\mathrm{q}_{\mathrm{ij}} \quad$ Element in confusion matrix normalized by fault proportion

$\mathrm{v}_{\mathrm{ij}} \quad$ Element in normalized cost matrix

$\mathrm{z} \quad$ Cumulative standard normal distribution

$\alpha \quad$ Significance level of the test (1-Confidence/100)

$\beta \quad$ Constant in calculation of normalized cost matrix

$\lambda \quad$ Laplace corrector

Operators

- Element by element matrix multiplication

- Boolean multiplication

\section{COST AND CONFUSION MATRICES}

The use of confusion matrices to demonstrate the ability of a diagnostic algorithm to differentiate faults is widespread and well understood. Table 1 presents a sample confusion matrix. The diagonal (in grey) represents faults correctly identified and the off diagonals represent faults misclassified. The no fault case can either be retained or removed from the matrix. More details on varieties of confusion matrices and metrics available to summarize them are given by Davison and Bird [1].

Table 1: Multiple fault confusion matrix

\begin{tabular}{|l|l|l|l|l|l|}
\hline & No-fault & F1 & F2 & F3 & F4 \\
\hline No-fault & A & B & C & D & E \\
\hline F1 & F & G & H & I & J \\
\hline F2 & K & L & M & N & O \\
\hline F3 & P & Q & R & S & T \\
\hline F4 & U & V & W & X & Y \\
\hline $\begin{array}{l}\text { T } \\
\text { Predicted State }\end{array}$
\end{tabular}

\section{Correcting for Fault Distribution}

Often, during development, testing and evaluation, fault distributions are assumed that are unrepresentative of what is experienced in service. This may be due to lack of knowledge of the true conditions or limitations in testing. Even fault distributions obtained from in-service data will change with the operating environment and duty cycle of the engines.

To correct for changes in fault distributions the original distribution must first be eliminated from the confusion matrix. This is achieved by dividing each element by the sum of the entries in the column containing it, as given by equation 1 . The new distribution is then applied by multiplying each column by the corresponding element in the fault probability vector as per equation 2. The fault probability vector contains the proportion of faults in element $\mathrm{j}$ corresponding to the column $\mathrm{j}$ in the 
confusion matrix. The sum of the elements in the fault probability vector is 1 .

$$
\begin{gathered}
q_{i j}=\frac{p_{i j}}{\sum_{k=1}^{F} p_{k j}} \\
p_{i j}^{\prime}=q_{i j} f_{j}
\end{gathered}
$$

Equation 2 produces a new confusion matrix with a distribution representative of the one expected in service. Unfortunately, algorithm validation with actual performance data is often very limited, due to the expense of operating and degrading an engine. Small sample sizes from fault implantation studies can result in large confidence intervals on the results.

Bootstrap techniques can be applied to obtain the confidence intervals on the confusion matrices and their corresponding metrics. This can be used to guide the number of samples required to obtain an acceptable confidence interval. Details on applying bootstrap techniques to diagnostic metrics can be found in Davison and Bird [1] and the general application and theory in Davison and Hinkley [7].

\section{Cost Matrix}

The confusion matrix adequately describes the ability of the algorithm to discriminate faults, and, if it has been adjusted for the expected fault distribution, should be representative of its performance in service. However, the confusion matrix does not allow for the costs involved with each element of the confusion matrix. The cost matrix is identical in size to the confusion matrix, with each corresponding element representing the cost of that outcome in the confusion matrix. If a profit is generated by the outcome the value is negative. The utility matrix is equivalent to the cost matrix, but provides the benefit or profit of each element in the confusion matrix. It is simply the negative of the cost matrix. The cost matrix is more appropriate for diagnostics analysis as no profit is expected and the user's objective is to minimize the expense incurred.

The cost of each diagnostic outcome will depend on both the application and user. Orsagh et al. [8], and Osborn and Yu [9] provide some of the costs associated with gas turbine diagnostic systems and their misdiagnosis. In general, if the cost of the diagnostic system is considered a constant and not incorporated as an outcome of the diagnosis then a correct diagnosis of no fault will cost nothing as the aircraft continues to function as planned and no action is required. The correct diagnosis of a fault incurs the cost of both investigation and repair. The incorrect diagnosis of a fault incurs the cost of investigation to determine that no fault is occurring.

The incorrect diagnosis of no fault has potentially the highest cost as it could include unscheduled down time and secondary damage to the engine. The incorrect isolation of the fault type will incur additional investigation costs. The value of a diagnostic algorithm is not truly known without incorporating these costs into the metrics.

The mean cost matrix combines the cost matrix and confusion matrix, essentially resulting in a confusion matrix weighted by the costs. The mean cost matrix is produced by performing an element wise multiplication of the cost matrix and the confusion matrix as per equation 3 .

$$
\mathbf{D}=\mathbf{C} \bullet \mathbf{P}
$$

As with the confusion matrix, the resulting mean cost matrices can be difficult to compare as they contain many values. The obvious solution is to sum the mean cost matrix as per equation 4. This will yield the mean cost per diagnosis, under the fault distribution assumed for the generation of the confusion matrix. A change in the fault distribution can have a very significant effect on the mean total cost. The value in has little meaning across different applications and users due to the variations in fault distribution and costs.

$$
\text { Mean Total Cost }=\sum_{i=1}^{F} \sum_{j=1}^{F} d_{i j}
$$

The mean subjective cost score (MSC) is proposed as an alternative to the simple mean total cost. It was developed by McDonald [10] and is normalized such that it returns a 0 when all faults are correctly classified and 1 when the faults are evenly distributed among all possible outcomes. It is calculated by equation 5 . The normalized cost matrix $(\mathbf{V})$ is found by rescaling the cost matrix as per equation 6 . The beta coefficients in equation 6 are obtained by simultaneously solving equations 7 and 8 , which satisfy the limits 0 and 1 as specified above.

$$
\begin{gathered}
M S C=1-\sum_{i=1}^{F} \sum_{j=1}^{F} p_{i j} v_{i j} \\
\mathbf{V}=\beta_{2}-\beta_{1} \mathbf{C} \\
1=\beta_{2}-\beta_{1}\left(\sum_{i=1}^{F} f_{i} c_{i i}\right) \\
0=\beta_{2}-\beta_{1}\left(\frac{1}{F} \sum_{i=1}^{F} f_{i} \sum_{j=1}^{F} c_{j i}\right)
\end{gathered}
$$

If the costs are assumed to be equal for all correct diagnoses, and equal, but greater than the correct cost, for misdiagnoses the MSC reduces to a metric for the confusion matrix alone, given by equation 9 . Other standard confusion 
matrix metrics, such as the Kappa coefficient, could also be applied to the mean cost matrix to obtain a metric for the cost.

$$
M S C=\frac{F}{F-1} \sum_{i=1}^{F} \sum_{j=1, j \neq i}^{F} p_{i j}
$$

While the mean total cost has the most direct relation to the in-service cost, and is useful when examining an algorithm for a particular application, the MSC is applicable across applications with the same relative cost differentials, but not necessarily the same absolute costs. The reporting of MSC and the normalized cost matrix also allows the merit of an algorithm to be demonstrated without revealing true operating costs, much as a normalized performance map provides compressor operating trends without revealing the actual performance parameters.

\section{Laplace Correction for Missing Data}

Test data sets do not usually contain a complete representation of the confusion matrix. The low probability of the off diagonal elements occurring requires a very large data set to accurately capture the true distribution of the complete population. Applying a Laplace correction to the mean cost matrix compensates for the missing data and improves the confidence interval on the resulting cost value [11].

In addition to improving the confidence interval on the average metric, we apply the Laplace correction to obtain improved confidence intervals on the entries in the confusion matrix, including zero value entries that otherwise would not have a confidence interval. Many zero values occur during an algorithm test program since enough faults cannot be implanted to capture all the possible misdiagnoses. It is impossible to know which, if any of the zero entries, would have a non-zero value in the complete population. A confidence interval can be assigned, however, to provide an indication of the variability.

The Laplace corrected matrix is produced with equation 10. Practically, this has the effect of adding $\lambda$ events to every cell in the confusion matrix. Whereas the usual assumption is an initial zero distribution in the confusion matrix, the Laplace correction assumes a uniform non-zero distribution (equal to the $\lambda$ value in equation 10 ). Increasing the value in the confusion matrix element decreases the effect of the Laplace correction. Increasing sample size also decreases the effect of the correction.

Margineantu and Dietterich [11] show that when calculating the overall cost a Laplace correction factor $(\lambda)$ between 0 and 0.5 improves the confidence interval for the bootstrap technique, bringing the confidence closer to the stated value. They did not, however, examine the confidence intervals for individual entries in the confusion matrix.

$$
\mathbf{L}=\frac{\mathbf{P} n+\lambda}{n+F^{2} \lambda}
$$

Our work shows that the Laplace correction also improves the confidence intervals on the individual entries in the confusion matrix. Starting with the confusion matrix presented in Table 2, which contains a range of proportions representing the true occurrence rates in a population, new matrices with sample sizes of: 20, 40, 80, 200 and 1000 were produced. 1000 matrices were generated for each sample size and the confidence intervals generated. The fraction of the confidence intervals containing the value from the original matrix divided by the confidence level should equal one. If it is less than one the confidence interval is too small and greater than one it is to large.

Table 2: Laplace test confusion matrix

\begin{tabular}{|l|l|l|l|l|}
\hline & \multicolumn{1}{|c|}{$\mathbf{A}$} & \multicolumn{1}{c|}{$\mathbf{B}$} & \multicolumn{1}{c|}{$\mathbf{C}$} & \multicolumn{1}{c|}{$\mathbf{D}$} \\
\hline $\mathbf{a}$ & 0.5 & 0.0002 & 0.001 & 0.01 \\
\hline $\mathbf{b}$ & 0.05 & 0.2 & 0.0001 & 0.001 \\
\hline $\mathbf{c}$ & 0.005 & 0.02 & 0.1 & 0.0002 \\
\hline $\mathbf{d}$ & 0.0005 & 0.002 & 0.01 & 0.1 \\
\hline
\end{tabular}

Figure 1 plots the fraction of confidence intervals that contain the true value across a range of $\lambda$ values for a sample size of 20. The confidence intervals, for all but the 0.1 proportion, are far too small without the Laplace correction. Despite starting at different fractions the small proportions all reach the true confidence level (y axis equals 1) at nearly the same Laplace correction of 0.035. After this point the fraction quickly increases to a value of 1.05 , where the confidence interval always contains the true value. Figure 2 is a similar plot for a sample size of 200. Increasing the sample size by a factor of 10 has shifted the curves up. The 0.01 proportion curve matches the 0.1 curve in Figure 1, the 0.001 matches the 0.01 curve and so on. The curves, however, still reach one at nearly the same Laplace correction value.

The optimal Laplace correction does changes with confidence interval, however. Figure 2 includes a curve for the $90 \%$ confidence level. Although it begins at the same level as the corresponding $95 \%$ confidence curve it does not begin to climb until much later. The optimal $\lambda$ value at the $90 \%$ level is 0.063 almost double that required at the $95 \%$ confidence level. Further work is required to fully define the optimal Laplace correction values at various sample sizes, expected values and confidence levels. 


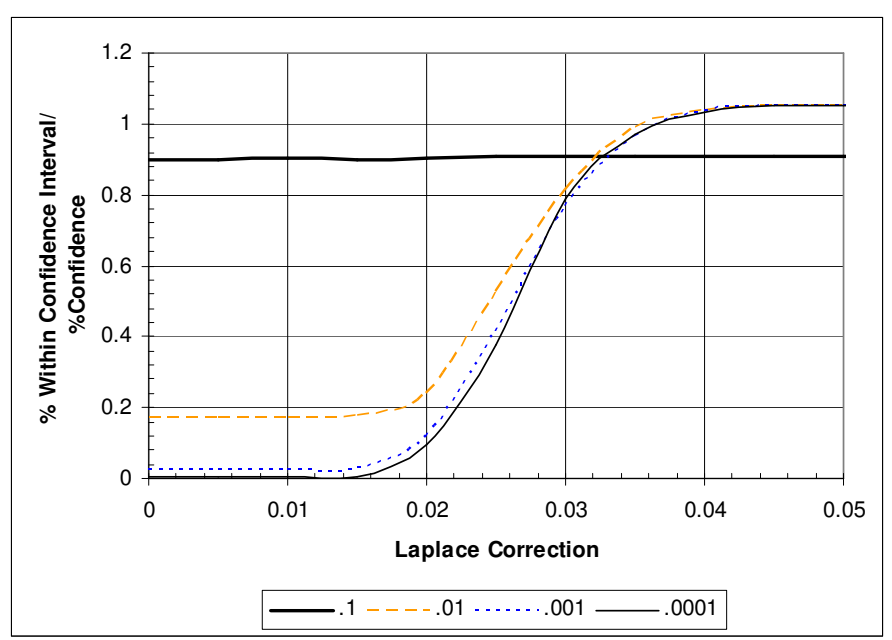

Figure 1: Accuracy of $95 \%$ confidence intervals with sample size of 20

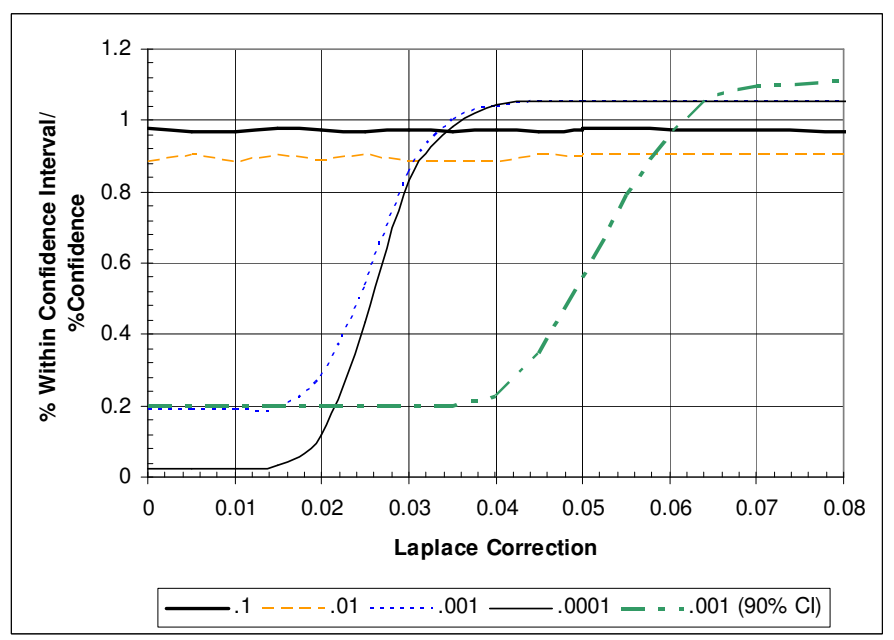

Figure 2: Accuracy of $95 \%$ confidence intervals with sample size of 200

\section{Example}

To demonstrate the utility of these functions an example case will be presented. The data are entirely notional but labels relevant to a gas turbine diagnostic system have been attached. The full data set consisted of 440 operating points where a fault occurred:

\section{Bleed Valve Fault $\left(\mathrm{F}_{\mathrm{B}}\right)-238$ occurrences Compressor fault $\left(\mathrm{F}_{\mathrm{C}}\right)-50$ occurrences LP Turbine fault $\left(\mathrm{F}_{\mathrm{L}}\right)-96$ occurrences HP Turbine fault $\left(\mathrm{F}_{\mathrm{H}}\right)-56$ occurrences}

Sample data sets, which might be generated from a fault implantation study, where simulated based on the full data set. Equal numbers of each fault were implanted, producing an even fault distribution. The algorithm was assumed to behave the same in the test and service environments and, therefore, the fault diagnoses were randomly selected from the set of diagnoses for the corresponding fault in the full operating data set. Sample sizes of 5, 10 and 20 for each fault were generated. The larger samples included the data from the smaller samples, as if the larger data set was being built on the smaller, as would be expected in an actual test program.

Increasing the number of implanted faults decreases the confidence interval on the resulting metrics. While not surprising, the confidence intervals for test data sets are often not presented. The method of applying the bootstrap is given by Davison and Bird [1]. For this example 1000 bootstrap samples were taken and a further 200 bootstrap samples per sample taken, to obtain the variance.

The bootstrap technique allows a confidence interval to be produced. Alternately, it can be used to determine the number of fault implantations required to obtain the desired confidence interval. Although cost is often the driving force behind the study size, this allows the cost benefit of increasing the sample size to be examined, and could even result in a decision to reduce the sample size.

Figure 3 demonstrates the change in confidence interval and the value of the MSC as the number of implanted faults is increased. Equation 9 was used to calculate the MSC. The results from the Laplace corrected data set are also shown. A lambda value of 0.25 was chosen because Margineantu and Dietterich [11] found this came close to achieving a true $95 \%$ confidence interval and erred by producing a larger confidence interval than required, resulting, in a conservative result. For comparison the result from the original data set, adjusted to match the implanted fault distribution, is also presented. Variation can be very significant for small numbers of implanted faults since the chance of having a sample from all the possible off diagonal combinations is low.

The confidence interval from the Laplace corrected matrix always contains the MSC for the full data set. The values for the uncorrected data are not as well behaved and at the 5 sample level the confidence interval does not intersect the confidence interval for the full data set. Similar patterns were found for the Kappa coefficient and percent correct classification (PCC). 


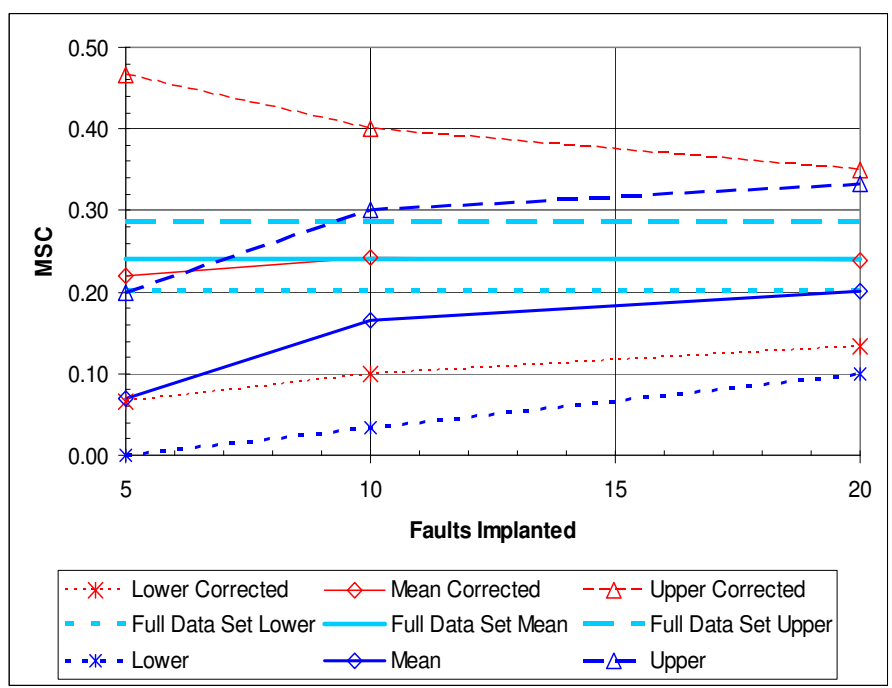

Figure 3: Confidence intervals on MSC for Laplace corrected $(\lambda=0.25)$ and original confusion matrices produced with increasing number of implanted faults

Figure 4 compares the size of the confidence interval for each sample size, both for the evenly distributed fault implantation and with the fault implantation rates adjusted to represent the fault distribution in the full data set. The Laplace corrected confidence intervals show a consistent decrease in range with sample size, as we would expect.

The uncorrected data displays more erratic behavior. The confidence interval initially increases in size because the increase in samples from five to ten introduces more variability into the data set than is compensated for by the larger sample size. The increase demonstrates that the confidence interval at the 5 sample size is too small. The introduction of the Laplace correction establishes a more representative variability in the very small sample sizes. As the sample size increases, the influence of the correction decreases and the sizes of confidence intervals converge.

This demonstrates the caution required when dealing with small sample sizes. For the bootstrap technique to accurately predict the variance and confidence interval the sample must be representative of the population. If the off diagonal elements in the confusion matrix are small then the chances of all possibilities being represented in the implanted fault set are low. This reduces the overall variability in the data set and consequently the confidence interval.

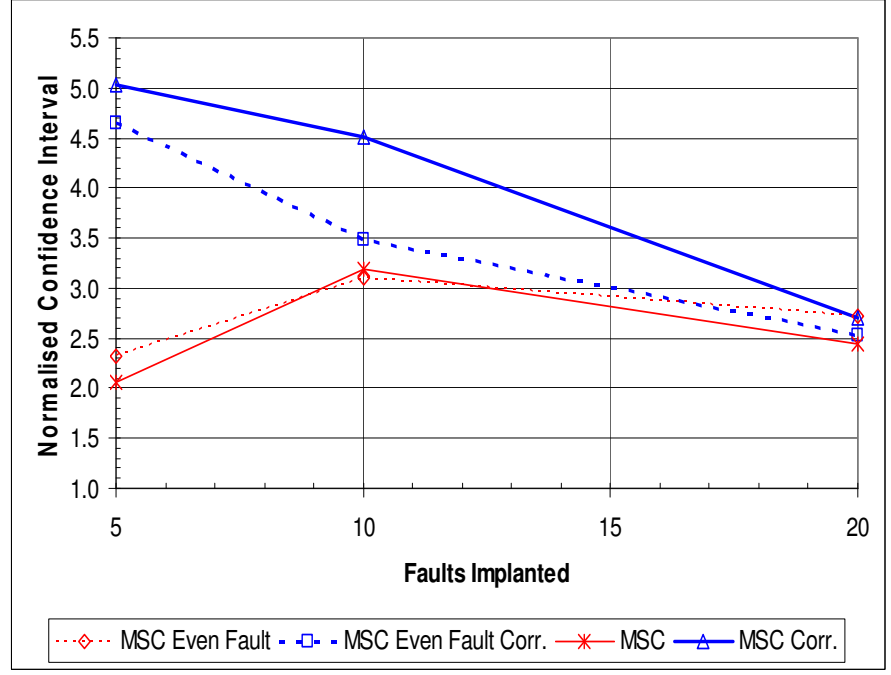

Figure 4: MSC confidence interval for Laplace corrected $(\lambda=0.25)$ and uncorrected confusion matrices normalized by confidence interval for the full data set

Comparing Tables 3 through 5 further demonstrates the small sample size problem. Tables 3 and 4 are the confusion matrices for a data set with 10 of each type of fault implanted. Table 5 is the confusion matrix for the full data set, adjusted for even fault distribution. Using element $(1,1)$ as an example the adjustment is made as follows:

1. As per equation 1 calculate $\mathrm{q}_{11}$ :

$q_{11}=\frac{p_{11}}{\sum_{k=1}^{F} p_{k 1}}=\frac{0.477}{0.477+0.041+0+0.023}=0.882$

2. As per equation 2 calculate the adjusted value. For even fault distribution the new distribution is $f=1 / F$.

$$
p_{11}^{\prime}=q_{11} f_{1}=\frac{q_{11}}{F}=\frac{0.882}{4}=0.221
$$

The bold zero values in Table 3 and 4 correspond to the small, but non-zero, bold values in Table 5. The lack of an example in the implanted data set results in no variance or confidence interval being determined for that value. The bootstrap technique assumes it is always zero, which the full data set shows is incorrect.

Applying a Laplace correction on the confusion matrix resulted in the intervals on the zero values given in Table 4. A $\lambda$ value of 0.035 was chosen because our tests showed this produced near 95\% confidence intervals across a range of proportions. The optimal correction factor is different when calculating confidence intervals on individual confusion matrix entries and when looking at aggregate metrics for the entire matrix. The Laplace correction is applied to the matrix and the 
bootstrap samples are then drawn from the proportions in the corrected matrix. Using element $(1,2)$, a zero value, in Table 3 as an example the Laplace correction is made by applying equation 10. The zero value is now a small value that can be sampled. This increase is compensated for by a reduction in the larger values. For example element $(1,1)$ goes from 0.200 to 0.198 .

$$
l_{12}=\frac{p_{12} n+\lambda}{n+F^{2} \lambda}=\frac{0(40)+0.035}{40+4^{2}(0.035)}=0.0009
$$

Table 3: Confusion matrix generated with 10 faults implanted for each fault type including $95 \%$ confidence interval

\begin{tabular}{|c|c|c|c|c|c|c|c|c|c|}
\hline & \multicolumn{2}{|c|}{$\overline{F_{B}}$} & \multicolumn{2}{|c|}{$\mathbf{F}_{\mathbf{C}}$} & \multicolumn{2}{|c|}{$\mathbf{F}_{\mathrm{L}}$} & \multicolumn{2}{|c|}{$\mathbf{F}_{H}$} & Total \\
\hline $\mathbf{F}_{\mathbf{B}}$ & 0.200 & $\begin{array}{l}0.350 \\
0.100\end{array}$ & 0 & NA & 0 & NA & 0.025 & \begin{tabular}{|l|}
0.075 \\
0.000
\end{tabular} & 0.225 \\
\hline $\mathbf{F}_{\mathbf{C}}$ & 0.050 & $\begin{array}{l}0.125 \\
0.000\end{array}$ & 0.250 & $\begin{array}{l}0.400 \\
0.125 \\
\end{array}$ & 0 & NA & $\mathbf{0}$ & NA & 0.300 \\
\hline $\mathbf{F}_{\mathbf{L}}$ & 0 & NA & 0 & NA & 0.225 & \begin{tabular}{|l|}
0.350 \\
0.100 \\
\end{tabular} & 0.025 & \begin{tabular}{|l|}
0.075 \\
0.000 \\
\end{tabular} & 0.250 \\
\hline $\mathbf{F}_{\mathbf{H}}$ & $\mathbf{0}$ & NA & 0 & NA & 0.025 & \begin{tabular}{|l|}
0.075 \\
0.000 \\
\end{tabular} & 0.200 & \begin{tabular}{|l|}
0.325 \\
0.100 \\
\end{tabular} & 0.225 \\
\hline Total & 0.2 & & 0.2 & & 0.2 & & 0.2 & & 0.875 \\
\hline
\end{tabular}

Table 4: Confusion matrix generated with 10 faults implanted for each fault type with Laplace corrected $\mathbf{9 5 \%}$ confidence interval $(\lambda=\mathbf{0 . 0 3 5})$

\begin{tabular}{|c|c|c|c|c|c|c|c|c|}
\hline & \multicolumn{2}{|c|}{$\overline{F_{B}}$} & \multicolumn{2}{|c|}{$\overline{\mathbf{F}_{\mathrm{C}}}$} & \multicolumn{2}{|c|}{$\mathbf{F}_{\mathrm{L}}$} & \multicolumn{2}{|c|}{$\mathbf{F}_{\mathbf{H}}$} \\
\hline $\mathbf{F}_{\mathbf{B}}$ & 0.200 & \begin{tabular}{|l|}
0.325 \\
0.100 \\
\end{tabular} & 0 & $\begin{array}{l}0.025 \\
0.000\end{array}$ & 0 & \begin{tabular}{|l|}
0.025 \\
0.000 \\
\end{tabular} & 0.025 & $\begin{array}{l}0.075 \\
0.000 \\
\end{array}$ \\
\hline $\mathbf{F}_{\mathbf{C}}$ & 0.050 & \begin{tabular}{|l|}
0.125 \\
0.000 \\
\end{tabular} & 0.250 & $\begin{array}{l}0.400 \\
0.125\end{array}$ & 0 & \begin{tabular}{|l|}
0.025 \\
0.000 \\
\end{tabular} & 0 & $\begin{array}{l}0.025 \\
0.000 \\
\end{array}$ \\
\hline $\mathbf{F}_{\mathbf{L}}$ & 0 & $\begin{array}{l}0.025 \\
0.000\end{array}$ & 0 & $\begin{array}{l}0.025 \\
0.000\end{array}$ & 0.225 & $\begin{array}{l}0.350 \\
0.100\end{array}$ & 0.025 & $\begin{array}{l}0.075 \\
0.000\end{array}$ \\
\hline $\mathbf{F}_{\mathbf{H}}$ & $\mathbf{0}$ & \begin{tabular}{|l|}
0.025 \\
0.000 \\
\end{tabular} & 0 & $\begin{array}{l}0.025 \\
0.000\end{array}$ & 0.025 & \begin{tabular}{|l|}
0.075 \\
0.000 \\
\end{tabular} & 0.200 & $\begin{array}{l}0.325 \\
0.075\end{array}$ \\
\hline
\end{tabular}

Table 5: Confusion matrix generated from full data set adjusted for even fault distribution including Laplace corrected $95 \%$ confidence interval $(\lambda=0.035)$

\begin{tabular}{|c|c|c|c|c|c|c|c|c|c|}
\hline & \multicolumn{2}{|c|}{$\mathbf{F}_{\mathbf{B}}$} & \multicolumn{2}{|c|}{$\mathbf{F}_{\mathrm{C}}$} & \multicolumn{2}{|c|}{$\mathbf{F}_{\mathrm{L}}$} & \multicolumn{2}{|c|}{$\mathbf{F}_{\mathbf{H}}$} & Total \\
\hline $\mathbf{F}_{\mathbf{B}}$ & 0.221 & \begin{tabular}{|l|}
0.264 \\
0.186
\end{tabular} & 0.055 & $\begin{array}{l}\text { 0.077 } \\
\mathbf{0 . 0 3 4}\end{array}$ & 0 & $\begin{array}{l}0.002 \\
0.000\end{array}$ & 0.013 & $\begin{array}{l}0.025 \\
0.005\end{array}$ & 0.289 \\
\hline $\mathbf{F}_{\mathbf{C}}$ & 0.019 & \begin{tabular}{|l}
0.032 \\
0.007 \\
\end{tabular} & 0.190 & \begin{tabular}{|l}
0.227 \\
0.155 \\
\end{tabular} & 0 & \begin{tabular}{|l|}
0.002 \\
0.000 \\
\end{tabular} & 0.013 & \begin{tabular}{|l|}
0.025 \\
0.005 \\
\end{tabular} & 0.222 \\
\hline $\mathbf{F}_{\mathbf{L}}$ & 0 & $\begin{array}{l}0.002 \\
0.000 \\
\end{array}$ & 0.005 & \begin{tabular}{|l|l}
0.014 \\
0.000 \\
\end{tabular} & 0.240 & \begin{tabular}{|l|}
0.282 \\
0.202 \\
\end{tabular} & 0.054 & \begin{tabular}{|l|}
0.075 \\
0.032 \\
\end{tabular} & 0.298 \\
\hline $\mathbf{F}_{\mathbf{H}}$ & 0.011 & \begin{tabular}{|l|}
0.020 \\
0.002
\end{tabular} & 0 & $\begin{array}{l}0.002 \\
0.000\end{array}$ & 0.010 & \begin{tabular}{|l|}
0.020 \\
0.002
\end{tabular} & 0.170 & \begin{tabular}{|l|}
0.209 \\
0.134
\end{tabular} & 0.191 \\
\hline Total & 0.2 & 50 & 0.2 & & 0.2 & & 0.2 & & 0.820 \\
\hline
\end{tabular}

Presenting the confusion matrix and the corresponding summary metrics for even fault distribution allows easy discrimination of the algorithm's ability to identify a particular fault. Normalizing each column by the fault frequency generates a matrix with a diagonal of ones for perfect fault discrimination. However, this does not convey all the important information. The actual fault proportion is a critical parameter in the overall effectiveness of the algorithm. As an extreme example, $100 \%$ diagnosis of a fault which never occurs is no use.

The effect of the fault distribution on the resulting summary metrics is demonstrated in Table 6 . The result for the Laplace corrected matrix is given in brackets and differs only slightly from the uncorrected value, as the sample size is large enough to reduce the significance of the correction. The MSC was calculated with equation 5. The calculation of PCC, kappa and confidence of rejection with a detailed example are provided by Davison and Bird [1]. With a high level of confidence the results for the even fault distribution present an inferior picture of the algorithms performance compared to the results based on the actual fault distribution.

Similarly, the cost of diagnosis or miss-diagnosis can strongly affect the overall value of an algorithm to the operator. Using the cost matrix presented in Table 7, the MSC was recalculated and is shown under "Adjusted for Cost" in Table 6. The addition of the cost data significantly reduced the MSC once again. The evenly distributed data dropped from 0.24 to 0.18 while the distributed data showed a smaller change going from 0.19 to 0.16 . This provides a $21 \%$ confidence level that the MSC has improved.

Comparing the original evenly distributed data without cost to the actual fault distribution with cost, we are almost 99\% confident the MSC has improved. If only the evenly distributed data had been examined, a superior algorithm for the desired application could be rejected.

In this example, the cost has a slightly larger effect than the fault distribution on the MSC. Based on this limited sample, applying the cost matrix will improve the result even if the fault distribution is not representative of operational reality. We can also compare the mean total cost. For the evenly distributed case, this results in a cost of 18.9 and of 13.2 for the actual fault distribution (these correspond to the MSCs of 0.18 and 0.16 ). 
Table 6: MSC, PCC and Kappa with $95 \%$ confidence intervals and confidence for rejection of Actual Distribution without Cost, being greater than value. Results in parenthesis have Laplace correction applied $(\lambda=0.25)$

\begin{tabular}{|c|c|c|c|c|c|}
\hline & \multicolumn{2}{|c|}{ No Cost Adjustment } & \multicolumn{2}{|c|}{ Adjusted for Cost } \\
\hline & & Even Dist. & $\begin{array}{c}\text { Actual } \\
\text { Dist. }\end{array}$ & Even Dist. & $\begin{array}{c}\text { Actual } \\
\text { Dist. }\end{array}$ \\
\hline \multirow{4}{*}{ MSC } & Value & 0.24 & 0.19 & 0.18 & 0.16 \\
\hline & Lower & $0.19(0.20)$ & $0.15(0.15)$ & $0.14(0.15)$ & $0.12(0.12)$ \\
\hline & Upper & $0.29(0.29)$ & $0.23(0.24)$ & $0.23(0.23)$ & $0.21(0.22)$ \\
\hline & \begin{tabular}{|l|} 
Conf. \\
\end{tabular} & $95 \%(94 \%)$ & & $45 \%(44 \%)$ & $21 \%(16 \%)$ \\
\hline \multirow{4}{*}{ PCC } & \begin{tabular}{|l|} 
Value \\
\end{tabular} & 0.82 & 0.86 & \multirow{6}{*}{\multicolumn{2}{|c|}{$\begin{array}{l}\text { Confidence of } \\
\text { rejection that even } \\
\text { dist., no cost MSC > } \\
\text { Actual dist., cost } \\
\text { adjusted MSC }\end{array}$}} \\
\hline & Lower & $0.79(0.78)$ & $0.83(0.82)$ & & \\
\hline & Upper & $0.86(0.85)$ & $0.89(0.24)$ & & \\
\hline & \begin{tabular}{|l|} 
Conf. \\
\end{tabular} & $5 \%(6 \%)$ & & & \\
\hline \multirow{4}{*}{ Kappa } & Value & 0.76 & 0.78 & & \\
\hline & Lower & $0.71(0.70)$ & $0.73(0.73)$ & & \\
\hline & Upper & $0.81(0.80)$ & $0.83(0.82)$ & \multirow{2}{*}{\multicolumn{2}{|c|}{$1.3 \%(1.2 \%)$}} \\
\hline & \begin{tabular}{|l|} 
Conf. \\
\end{tabular} & $27 \%(29 \%)$ & & & \\
\hline
\end{tabular}

Table 7: Cost matrix

\begin{tabular}{|l|l|l|l|l|}
\hline & $\mathbf{F}_{\mathbf{B}}$ & $\mathbf{F}_{\mathbf{C}}$ & $\mathbf{F}_{\mathbf{L}}$ & $\mathbf{F}_{\mathbf{H}}$ \\
\hline $\mathbf{F}_{\mathbf{B}}$ & 3 & 18 & 25 & 33 \\
\hline $\mathbf{F}_{\mathbf{C}}$ & 11 & 15 & 32 & 38 \\
\hline $\mathbf{F}_{\mathbf{L}}$ & 13 & 27 & 22 & 39 \\
\hline $\mathbf{F}_{\mathbf{H}}$ & 17 & 34 & 41 & 30 \\
\hline
\end{tabular}

\section{COST CURVES}

ROC curves are used to evaluate diagnostic system performance across a range of threshold settings. This allows the algorithm's performance to be assessed across a range of possible usage. Cost curves have been presented in the artificial intelligence community as an alternative to ROC curves [5,6]. Cost curves offer several advantages over ROC curves:

1) Misclassification costs and fault probabilities can be incorporated into the performance assessment.

2) For given costs and probabilities an algorithm can be readily compared to a trivial classifier.

3) The performance of two algorithms can easily be compared.

4) An average value for several evaluations of an algorithm can be found.

5) Confidence intervals can be readily generated.

The ROC curve is based on the decision matrix, for example Table 8 . The rates are found by dividing each element in a column by the sum of the column. This removes the fault distribution from the analysis. The false positive rate is then plotted on the $\mathrm{x}$-axis $(\mathrm{C} /(\mathrm{A}+\mathrm{C})$ in Table 8$)$ versus the true positive rate on the $\mathrm{y}$-axis $(\mathrm{D} /(\mathrm{D}+\mathrm{B}))$ across a range of threshold settings in the algorithm under evaluation.
Table 8: Decision matrix

\begin{tabular}{|c|c|c|c|}
\hline & No-fault & Fault & $\longleftarrow$ True State \\
\hline No-fault & A & B & $A+D=$ Proportion of cases \\
\hline Fault & $\mathrm{C}$ & $\mathrm{D}$ & diagnosed correctly \\
\hline \multirow{2}{*}{\multicolumn{3}{|c|}{ Predicted State }} & $\begin{array}{l}A=\text { True Negative Proportion } \\
B=\text { False Negative Proportion }\end{array}$ \\
\hline & & & $\begin{array}{l}C=\text { False Positive Proportion } \\
D=\text { True Positive Proportion }\end{array}$ \\
\hline
\end{tabular}

The cost curve replaces each point on the ROC curve with a line spanning the proportion of fault occurrence from zero to one. Using the element labels in Table 8 for both the decision matrix and the associated cost matrix the $\mathrm{x}$-axis for the cost curve is the probability of a fault occurring, times cost and normalized, referred to as the probability cost function (PCF) and defined as follows:

$$
\begin{aligned}
P C F & =\frac{p_{+} C_{B}}{p_{+} C_{B}+\left(1-p_{+}\right) C_{C}} \\
p_{+}= & \text {Proportion of positive (fault) } \\
& \text { cases assigned independent of actual } \\
& \text { proportion from decision matrix }
\end{aligned}
$$
by:

The $y$-axis is the normalized expected cost (NEC) given

$$
\begin{aligned}
N E C & =\frac{\frac{P_{B}}{P_{B}+P_{D}} p_{+} C_{B}+\frac{P_{C}}{P_{A}+P_{C}}\left(1-p_{+}\right) C_{C}}{p_{+} C_{B}+\left(1-p_{+}\right) C_{C}} \\
& =\frac{(1-T P) p_{+} C_{B}+F P\left(1-p_{+}\right) C_{C}}{p_{+} C_{B}+\left(1-p_{+}\right) C_{C}}
\end{aligned}
$$

For each point on the ROC curve two points on the line can be obtained by setting $\mathrm{p}_{+}$to 0 and 1 . If the misdiagnosis costs are equal but greater than the equal diagnosis costs (logical statement 13 is satisfied), then the plot is simplified. Equation 11 becomes equation 14, which is just the assigned probability of a fault occurring, and equation 12 becomes 15, which is the error rate.

$$
\begin{aligned}
& \text { if }\left(C_{A}=C_{D}\right) \circ\left(C_{B}=C_{C}\right) \circ\left(C_{B}>C_{A}\right) \text { then : } \\
& P C F=p_{+} \\
& \qquad \begin{aligned}
N E C= & \frac{P_{B}}{P_{B}+P_{D}} p_{+}+\frac{P_{C}}{P_{A}+P_{C}}\left(1-p_{+}\right) \\
= & (1-T P) p_{+}+F P\left(1-p_{+}\right)
\end{aligned}
\end{aligned}
$$


Figure 5 shows a sample ROC curve. The points labeled A and $\mathrm{F}$ represent the extreme, simple, classifiers. Point A assigns everything to no-fault and $\mathrm{F}$ to fault. These are converted to cost curve lines A and F in Figures 6 and 7. Anything under the triangle formed by lines A and $\mathrm{F}$ is superior to using the simple classifiers; anything that falls above lines is inferior. Any classifier resulting in a NEC above 0.5 will be improved by just swapping the labels. In other words, any case classified as a fault should be classified as no-fault and vice-versa.

Line D in Figure 6 corresponds to point D in Figure 5 and we can immediately see the range of PCF for which the algorithm, with the appropriate threshold settings to achieve point $\mathrm{D}$, outperforms the simple classifiers. The objective on the cost curve plot is to approach an expected cost of 0 , which equates to always getting the answer correct.

Figure 7 plots the cost curves for both the algorithms presented in Figure 5. The lines corresponding to the points on the "algorithm 1" ROC curve are presented and labeled. This cost curve was formed by tracing the lines with the smallest NEC. Forming the cost curve in this way produces the best case curve. Unfortunately, to achieve this curve in reality requires the proportion of faults and costs to be known prior to operation, so that the correct thresholds can be set. However, this plot provides guidance in determining the optimal threshold settings to maximize performance across an expected range of fault occurrence. It also allows poor settings to be isolated. Line B, for example, is always inferior to other settings as no segment of it forms part of the optimal cost curve.

A cost curve can be generated from a single decision matrix. This produces a single line and its performance across the range of costs can be assessed. The line can be compared to the lines produced for the decision matrices from other algorithms, or ones using different thresholds, to determine which is optimal for the expected cost for a particular application. The cost curve immediately shows under which costs each algorithm is superior and the cost at which it is beneficial to switch algorithms.

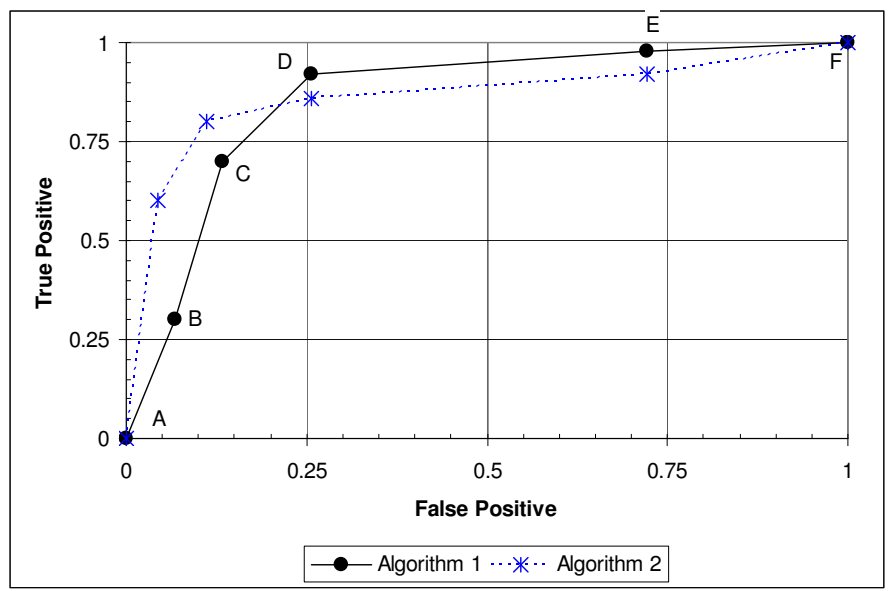

Figure 5: Sample ROC curve

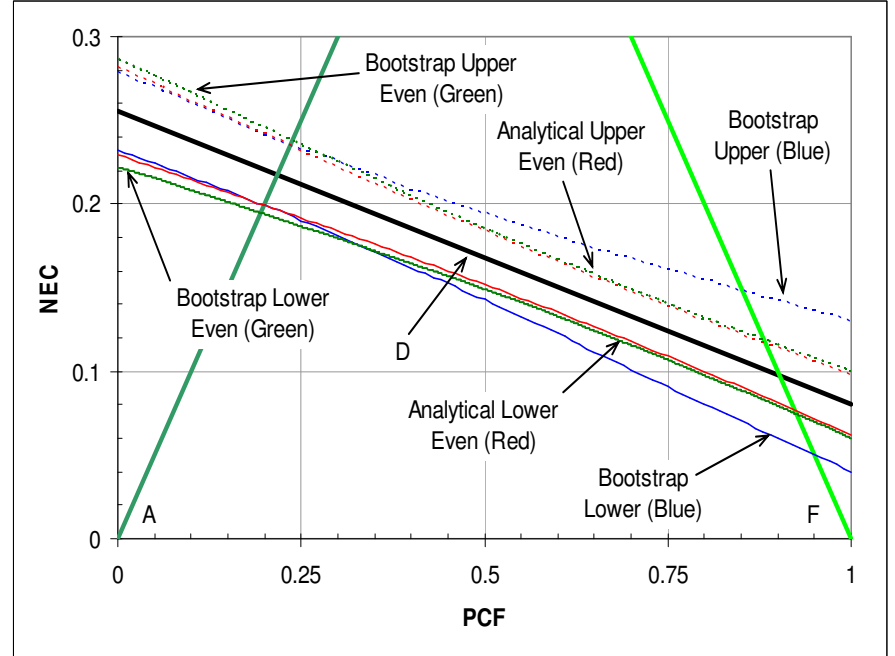

Figure 6: Sample cost curve for single threshold setting showing simple classifiers and $\mathbf{9 0 \%}$ confidence intervals

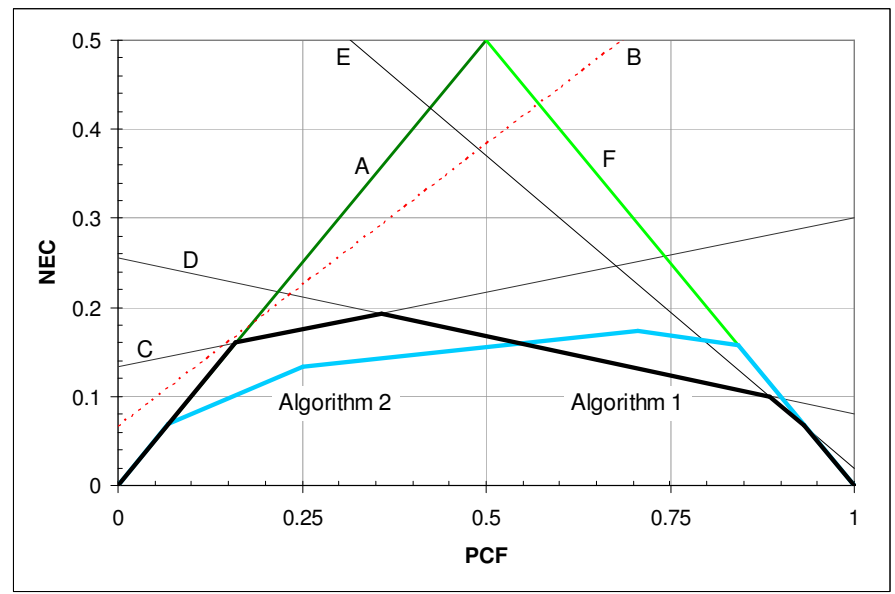

Figure 7: Cost curves for algorithm 1 and 2

\section{Summary of Cost Curve Production}

To produce a cost curve for a single decision matrix:

1. Set $\mathrm{p}_{+}=0$ resulting in a $\mathrm{PCF}=0$ (equation 11 ) and a $\mathrm{NEC}=\mathrm{FP}$ (equation 12)

2. Set $\mathrm{p}_{+}=1$ resulting in a $\mathrm{PCF}=1$ (equation 11 ) and an NEC $=1-\mathrm{TP}$ (equation 12)

3. Connect the two points produced above

The steps above can be repeated for each threshold setting. The best performance can be defined by tracing the line segments closest to NEC $=0$. For a given PCF the line with the lowest NEC will represent the optimal combination of algorithm and threshold settings. 


\section{Averaging and Confidence Intervals}

It is important to be able to use statistical techniques to improve the quality of data obtained either in service or during development. Perhaps the most basic technique is averaging. However, there is no agreement on the optimal technique for averaging ROC curves.

The cost curve can be averaged by taking the mean NEC for each PCF value. The average cost curve is then the average NEC, assuming that the optimal classifier settings have been used for the given PCF [6]. Once the average cost curve has been generated equations 11 and 12 can be inverted to find the true and false positive rates and produce an average ROC curve.

An analytical technique has been presented for generating a confidence interval at any point on the cost curve [12]. Equation 16 provides the variance. This assumes that the sample represents the fault distribution in the population and that the distribution of the diagnosis is Gaussian. Once the variance is known a confidence interval on the NEC can be found for any PCF value with equation 17.

$$
\begin{gathered}
s^{2}=P C F^{2}(1-T P) T P+(P C F-1)^{2} F P(1-F P) \\
U=N E C+\frac{s z_{\alpha / 2}}{\sqrt{n}} \\
L=N E C-\frac{s z_{\alpha / 2}}{\sqrt{n}}
\end{gathered}
$$

Applied to a single cost line, the result can be seen in Figure 6 , labeled as the analytical upper and lower confidence intervals. Alternately, a bootstrap technique can be applied. The decision matrix is sampled the required number of times to produce a set of cost lines. Stratified sampling was performed forcing each sample to have the same fault distribution as the original. This assumes that the sample represents the fault distribution in the population as with equation 16. At each point along the PCF axis, the upper and lower confidence interval cost lines are chosen as per equation 18. $\mathrm{n}_{\text {lower }}$ and $\mathrm{n}_{\text {upper }}$ are the position of the line in the set of bootstrap sampled lines, ordered from lowest to highest NEC, at the particular PCF.

$$
\begin{aligned}
& n_{\text {lower }}=n(\alpha / 2) \\
& n_{\text {upper }}=n(1-\alpha / 2) \\
& n=\text { number of bootstrap samples }
\end{aligned}
$$

The bootstrap confidence interval is also shown in Figure 6. As is typical in gas turbine diagnostics, the sample set has relatively few positive examples $(10 \%)$ resulting in a larger confidence interval as PCF approaches 1 , and the positive examples form the majority of the available data. The analytical technique was less successful at portraying this trend.

At low PCF values, the analytical and bootstrap are very similar since nearly all the samples are contributing to the NEC calculation. At higher PCF values, the number of samples contributing to the NEC calculation decreases and the bootstrap confidence interval spreads out to reflect this. If the decision matrix is based on a sample set with equal numbers of fault and no fault cases (even distribution), the confidence interval generated is very close to the analytical technique as shown in Figure 6.

If the bootstrap technique is applied to all the cost curve lines generating the optimal cost curve, an overall confidence interval can be generated. The result is shown in Figure 8. We can now see not only which algorithm is superior but have some idea of how much better it is. The effect of not using stratified sampling and hence not forcing the same fault distribution each time was examined and found to be insignificant with these data sets.

The analytical version of the confidence interval was also generated. This is a relatively simple process. The confidence interval was calculated at the intersection of the cost lines. As the variance depends on the true and false positive proportions the confidence intervals will be different for each line. We chose to use the largest confidence interval at each intersection and join the resulting points with straight lines. The results of the process are shown in Figure 8 for algorithm 1. As with the single cost line the confidence interval is underestimated at higher PCF values. Finally the confidence interval on the cost curves can be converted back to an ROC curve to produce a corresponding confidence interval.

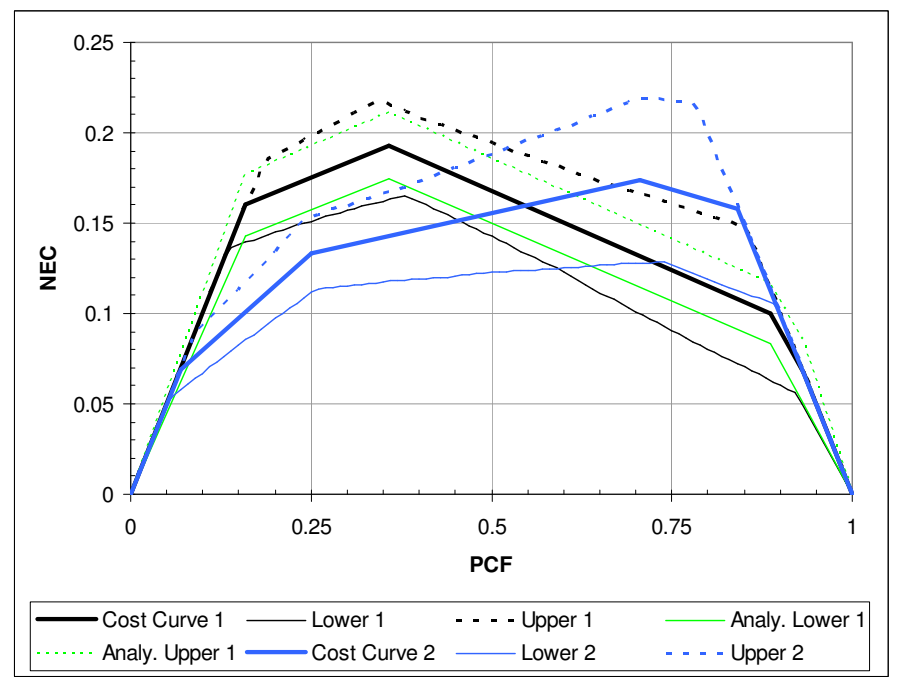

Figure 8: Cost curves showing bootstrap and analytical $\mathbf{9 0 \%}$ confidence intervals for original sample size of $\mathbf{1 0 0 0}$ 


\section{CONCLUSIONS}

Proper assessment of diagnostic algorithms requires incorporation of real world fault distributions and costs into the test data. Techniques to do this have been presented and their utility demonstrated.

Cost curves were explained and contrasted to the commonly used ROC curves. Cost curves have the advantage of providing an easy comparison between algorithms across a range of fault occurrences and costs.

Further work is required in determining the optimal Laplace correction for individual confusion matrix elements to obtain the desired confidence interval. The correction varies with confidence level and this needs to be examined.

\section{REFERENCES}

[1] Davison, C. R. and Bird, J. W., 2008, "Review Of Metrics And Assignment Of Confidence Intervals For Health Management Of Gas Turbine Engines", ASME GT200850849, ASME Turbo Expo 2008, June 2008, Berlin, Germany.

[2] Simon, D.L.; Bird, J.W.; Davison, C.R.; Volponi, A. and Iverson, R.E., 2008, "Benchmarking Gas Path Diagnostic Methods: A Public Approach", ASME GT2008-51360, ASME Turbo Expo 2008, June 2008, Berlin, Germany.

[3] DePold, H.; Siegel, J. and Hull, J., 2004, "Metrics for Evaluating the Accuracy of Diagnostic Fault Detection Systems", ASME GT2004-54144, IGTI Turbo Expo 2004, June 2004, Vienna, Austria.

[4] Webb, G.I. and Ting, K.M., 2005, "On the Application of ROC Analysis to Predict Classification Performance under Varying Class Distributions," Machine Learning, January 2005, Vol. 58 (1), pp.25-32.

[5] Drummond, C. and Holte, R.C., "Explicitly Representing Expected Cost: An Alternative to ROC Representation", Proceedings of KDD-2000, Sixth ACM SIGKDD International Conference on Knowledge Discovery and Data Mining, August 20-23, 2000, Boston, MA, pp.198207.

[6] Drummond, C. and Holte, R.C., "Cost curves: An improved method for visualizing classifier performance", Machine Learning, October 2006, Vol. 65 (1), pp. 95-130.

[7] Davison, A.C. and Hinkley, D.V., 1997, Bootstrap Methods and their Applications, Cambridge University Press, Cambridge, U.K.

[8] Orsagh, R.F.; Roemer, M.J.; Savage, C.J. and Lebold, M., "Development of Performance and Effectiveness Metrics for Gas Turbine Diagnostic Techniques," Aerospace, 2002 IEEE Conference Proceedings, 2002, Vol. 6, pp. 28252834.

[9] Osborn, M. and Yu, L.J., 2007, "Decision Support for Remote Monitoring and Diagnostics of Aircraft Engine Using Influence Diagrams," ASME GT2007-28331, IGTI Turbo Expo 2007, May 2007, Montreal, QC.

[10] McDonald, R.A., "The Mean Subjective Utility Score, A Novel Metric for Cost-Sensitive Classifier Evaluation", Pattern Recognition Letters, 2006, Vol. 27, pp. 1472-1477.
[11] Margineantu, D.D. and Dietterich, T.G., "Bootstrap Methods for the Cost-Sensitive Evaluation of Classifiers", Proceedings of the Seventeenth International Conference on Machine Learning, 2000, pp. 582-590.

[12]Dugas, C. and Gadoury, D., "Positive Exact Distributions of Cost Curves", Proceedings of the 25th International Conference on Machine Learning, Helsinki, Finland, 2008. 